\title{
On sums and products of residues modulo $p$
}

\author{
by
}

\section{A. SÁRKÖZY (Budapest)}

1. Introduction. Throughout the paper we use the notation $e(\alpha)=e^{2 \pi i \alpha}$.

Our goal is to show that if $A, B, C, D$ are "large" subsets of $\mathbb{Z}_{p}$, then the equation

$$
a+b=c d, \quad a \in A, b \in B, c \in C, d \in D,
$$

can be solved.

Theorem. If $p$ is a prime, $A, B, C, D \subset \mathbb{Z}_{p}$, and the number of solutions of (1) is denoted by $N$, then

$$
\left|N-\frac{|A||B||C||D|}{p}\right| \leq(|A||B||C||D|)^{1 / 2} p^{1 / 2} .
$$

Corollary 1. If $p$ is a prime, $A, B, C, D \subset \mathbb{Z}_{p}$ and

$$
|A||B||C||D|>p^{3}
$$

then (1) can be solved.

Note that Corollary 1 and thus also the Theorem is the best possible apart from the constant factor in (2), resp. (3). Indeed, taking $A=B=$ $\{n: 1 \leq n<p / 2\}$ (here and in what follows we do not distinguish between integers and residue classes represented by them), $C=\{1, \ldots, p\}$ and $D=$ $\{0\}$, we have

$$
|A||B||C||D|=\left(\frac{1}{4}+o(1)\right) p^{3},
$$

however, (1) has no solution.

Moreover, we remark that these results cannot be extended from prime moduli to composite moduli, i.e., from $\mathbb{Z}_{p}$ to $\mathbb{Z}_{m}$. Indeed, let $m=2 k$ be an even positive integer, and let $A=C=\{2,4, \ldots, 2 k\} \subset \mathbb{Z}_{m}, B=$ $\{1,3, \ldots, 2 k-1\}$ and $D=\mathbb{Z}_{m}$. Then we have

2000 Mathematics Subject Classification: 11Bxx, 11Txx.

Research partially supported by Hungarian National Foundation for Scientific Research, Grant No. T043623. 


$$
|A||B||C||D|=\frac{1}{8} m^{4}
$$

so that much more holds than the $m$-analogue of (3), however, clearly (1) has no solution. One might like to study the question that in what rings $R$ (including infinite ones) is it true that if $A, B, C, D$ are "dense" subsets of $R$, then (1) must be solvable.

First, in Section 2 we will show that the Theorem and Corollary 1 generalize several earlier theorems, and the proofs of the Theorem and Corollary 1 will be presented in Section 3.

\section{Consequences}

COROLlaRY 2. If $p$ is a prime number, $\chi$ is a (multiplicative) character modulo $p$ of order $d, n \in \mathbb{Z}, A, B \subset \mathbb{Z}_{p}$ and

$$
|A||B|>d^{2}\left(1-\frac{1}{p}\right)^{-2} p
$$

then there are $a \in A, b \in B$ with

$$
\chi(a+b)=e\left(\frac{n}{d}\right) .
$$

Proof. Writing $C=\left\{u: u \in \mathbb{Z}_{p}, \chi(u)=1\right\}$ and $D=\left\{v: v \in \mathbb{Z}_{p}, \chi(v)=\right.$ $\left.e\left(\frac{n}{d}\right)\right\}$, we have

$$
|C|=|D|=\frac{p-1}{d}
$$

so that, by (4),

$$
|A||B||C||D|>d^{2} \frac{p^{3}}{(p-1)^{2}} \frac{(p-1)^{2}}{d^{2}}=p^{3} .
$$

Thus by Corollary $1,(1)$ can be solved. If $a, b, c, d$ satisfy (1) then we have

$$
\chi(a+b)=\chi(c d)=\chi(c) \chi(d)=1 \cdot e\left(\frac{n}{d}\right)=e\left(\frac{n}{d}\right)
$$

so that (5) holds and this completes the proof of Corollary 2.

In particular, if $\chi(n)=\left(\frac{n}{p}\right)$ (for $(n, p)=1$ ) is the Legendre symbol in Corollary 2 so that $d=2$, then we have the following consequence:

Corollary 3. If $p$ is an odd prime, $A, B \subset \mathbb{Z}_{p}$ and

$$
|A||B|>4\left(1-\frac{1}{p}\right)^{-2} p,
$$

then there are $a, a^{\prime} \in A, b, b^{\prime} \in B$ with

$$
\left(\frac{a+b}{p}\right)=1, \quad\left(\frac{a^{\prime}+b^{\prime}}{p}\right)=-1 .
$$


This sharpens and generalizes a result of Erdős and Sárközy [1]; see also [2] and [3].

Corollary 4. If $p$ is a prime, $k \in \mathbb{N},(p-1, k)>1, A, B \subset \mathbb{Z}_{p}$ and for all $a \in A, b \in B, a+b$ is a kth power in $\mathbb{Z}_{p}$, i.e., writing $E=\left\{x^{k}: x \in \mathbb{Z}_{p}\right\}$ we have $A+B \subset E$, then

$$
|A||B| \leq 9\left(1-\frac{1}{p}\right)^{-2} p .
$$

Note that apart from the constant factor in the upper bound in (6), this is Gyarmati's Theorem 8(b) in [5].

Proof of Corollary 4. We have to show that if $A, B \subset \mathbb{Z}_{p}$ and

$$
|A||B|>9\left(1-\frac{1}{p}\right)^{-2} p
$$

then there are $a \in A, b \in B$ with

$$
a+b \notin E .
$$

Write $D=(p-1, k)$ (so that $D>1$ ), let $r(n, D)$ denote the least nonnegative residue of $n$ modulo $D$, let $g$ be a primitive root modulo $p$, and define $C, D$ by $C=\left\{g^{u}: 0 \leq r(u, D)<D / 2\right\}, D=\left\{g^{v}: 0<r(v, D) \leq\right.$ $D / 2\}$ so that, by $D>1$,

$$
\min \{|C|,|D|\} \geq\left[\frac{D}{2}\right] \frac{p-1}{D} \geq \frac{p-1}{3} .
$$

By (7) and (9) we have

$$
|A||B||C||D|>9\left(1-\frac{1}{p}\right)^{-2} p\left(\frac{p-1}{3}\right)^{2}=p^{3}
$$

so that, by Corollary $1,(1)$ can be solved. If $a, b, c, d$ satisfy (1) then $a+b$ can be written in form

$$
a+b=c d=g^{u} \cdot g^{v}=g^{u+v}
$$

with $0<r(u+v, D)<D$ so that $D \nmid(u+v)$. Thus $D$ does not divide the (base $g$ ) index of $a+b$ modulo $p$ whence (8) follows.

Corollary 5. If $p$ is a prime, $k \in \mathbb{N}, A, B \subset \mathbb{Z}_{p}$ and, writing $D=$ $(k, p-1)$, we have

$$
|A||B|>D^{2}\left(1-\frac{1}{p}\right)^{-2} p,
$$

then the equation

$$
a+b=x^{k}, \quad a \in A, b \in B, x \in \mathbb{Z}_{p}, x \neq 0,
$$

can be solved. 
This is a variant of a special case of Gyarmati's Theorem 10(b) in [5]. Note that it follows from this corollary that if $m, n, k \in \mathbb{N}$ are fixed and $p$ is a prime large enough then the congruence

$$
x^{m}+y^{n} \equiv z^{k}(\bmod p),
$$

and in particular the Fermat congruence

$$
x^{n}+y^{n} \equiv z^{n}(\bmod p)
$$

has non-trivial solution $x, y, z$; the latter is Schur's theorem [7].

Proof of Corollary 5. Writing $F=\left\{x^{k}: x \in \mathbb{Z}_{p}, x \neq 0\right\}$, we clearly have

$$
|F|=\frac{p-1}{D} \text {. }
$$

Thus taking $C=D=F$, by (10) we have

$$
|A||B||C||D|=|A||B|\left(\frac{p-1}{D}\right)^{2}>p^{3}
$$

so that by Corollary 1 (1) can be solved. For $a, b, c, d$ satisfying (1) we have

$$
a+b=c d \in C D=F \cdot F=F
$$

which proves the solvability of (11).

Corollary 6. If $p$ is a prime, $S, T$ are integers with $1 \leq T \leq p$, $C, D \subset \mathbb{Z}_{p}$ and

$$
|C||D|>\frac{4}{T^{2}} p^{3}
$$

then

$$
c d \equiv n(\bmod p), \quad c \in C, d \in D, S<n \leq S+T,
$$

can be solved.

This is a slight sharpening of the Corollary in [6]; the connection with the problem of the least quadratic non-residue was analyzed there. See also [4].

Proof of Corollary 6. Define $A, B$ by $A=\{a: S \leq a \leq S+[T / 2]\}$, $B=\{b: 0<b \leq T-[T / 2]\}$ so that

$$
\min \{|A|,|B|\} \geq T-\left[\frac{T}{2}\right] \geq \frac{T}{2} .
$$

It follows from (12) and (14) that

$$
|A||B||C||D|>\left(\frac{T}{2}\right)^{2} \frac{4}{T^{2}} p^{3}=p^{3}
$$

so that, by Corollary 1 , there are $a, b, c, d$ satisfying (1):

$$
a+b=c d \text {. }
$$


By the definition of $A$ and $B$, here we have

$$
S<a+b \leq S+T
$$

and (13) follows from (15) and (16).

\section{The proofs}

Proof of the Theorem. For every $a, b, c, d \in \mathbb{Z}_{p}$ we have

$$
\frac{1}{p} \sum_{k=0}^{p-1} e\left((a+b-c d) \frac{k}{p}\right)= \begin{cases}1 & \text { if } a+b=c d, \\ 0 & \text { if } a+b \neq c d,\end{cases}
$$

so that

$$
N=\frac{1}{p} \sum_{a \in A} \sum_{b \in B} \sum_{c \in C} \sum_{d \in D} \sum_{k=0}^{p-1} e\left((a+b-c d) \frac{k}{p}\right) .
$$

Separating the term with $k=0$ we obtain

$$
\begin{aligned}
& N=\frac{|A||B||C||D|}{p}+\frac{1}{p} \sum_{k=1}^{p-1} \sum_{a \in A} \sum_{b \in B} \sum_{c \in C} \sum_{d \in D} e\left((a+b-c d) \frac{k}{p}\right) \\
& =\frac{|A||B||C||D|}{p}+\frac{1}{p} \sum_{k=1}^{p-1}\left(\sum_{a \in A} e\left(a \frac{k}{p}\right)\right)\left(\sum_{b \in B} e\left(b \frac{k}{p}\right)\right)\left(\sum_{c \in C} \sum_{d \in D} e\left(-c d \frac{k}{p}\right)\right)
\end{aligned}
$$

whence, writing $F(\alpha)=\sum_{a \in A} e(a \alpha)$ and $G(\alpha)=\sum_{b \in B} e(b \beta)$,

$$
\begin{aligned}
|| N \mid- & \frac{|A||B||C||D|}{p} \mid \\
& =\frac{1}{p}\left|\sum_{k=1}^{p-1} F\left(\frac{k}{p}\right) G\left(\frac{k}{p}\right)\left(\sum_{c \in C} \sum_{d \in D} e\left(-c d \frac{k}{p}\right)\right)\right| \\
& \leq \frac{1}{p} \sum_{k=1}^{p-1}\left|F\left(\frac{k}{p}\right)\right|\left|G\left(\frac{k}{p}\right)\right|\left|\sum_{c \in C} \sum_{d \in D} e\left(-c d \frac{k}{p}\right)\right| .
\end{aligned}
$$

Now we need Vinogradov's lemma [8, p. 29]:

Lemma 7. Let $(a, q)=1, q>1$. Let

$$
S=\sum_{x=0}^{q-1} \sum_{y=0}^{q-1} \zeta(x) \eta(y) e\left(x y \frac{a}{q}\right)
$$

and suppose that

$$
\sum_{x=0}^{q-1}|\zeta(x)|^{2}=X_{0}, \quad \sum_{y=0}^{q-1}|\eta(y)|^{2}=Y_{0} .
$$


Then

$$
|S| \leq\left(X_{0} Y_{0} q\right)^{1 / 2}
$$

We use this lemma with $a=-k, q=p$,

$$
\zeta(x)=\left\{\begin{array}{ll}
1 & \text { if } x \in C, \\
0 & \text { if } x \notin C,
\end{array} \quad \eta(x)= \begin{cases}1 & \text { if } d \in D, \\
0 & \text { if } d \notin D,\end{cases}\right.
$$

so that $X_{0}=|C|$ and $Y_{0}=|D|$. We obtain

$$
\left|\sum_{c \in C} \sum_{d \in D} e\left(-c d \frac{k}{p}\right)\right| \leq(|C||D| p)^{1 / 2} \quad \text { for }(k, p)=1 .
$$

By using Cauchy's inequality and a Parseval formula type identity, it follows from (17) and (18) that

$$
\begin{aligned}
\left|N-\frac{|A||B||C||D| \mid}{p}\right| & \leq \frac{1}{p} \sum_{k=1}^{p-1}\left|F\left(\frac{k}{p}\right)\right|\left|G\left(\frac{k}{p}\right)\right|(|C||D| p)^{1 / 2} \\
& \leq \frac{(|C||D|)^{1 / 2}}{p^{1 / 2}} \sum_{k=0}^{p-1}\left|F\left(\frac{k}{p}\right)\right|\left|G\left(\frac{k}{p}\right)\right| \\
& \leq\left(\frac{|C||D|}{p}\right)^{1 / 2}\left(\sum_{k=0}^{p-1}\left|F\left(\frac{k}{p}\right)\right|^{2}\right)^{1 / 2}\left(\sum_{k=0}^{p-1}\left|G\left(\frac{k}{p}\right)\right|^{2}\right)^{1 / 2} \\
& =\left(\frac{|C||D|}{p}\right)^{1 / 2}(|A| p)^{1 / 2}(|B| p)^{1 / 2} \\
& =(|A||B||C||D|)^{1 / 2} p^{1 / 2}
\end{aligned}
$$

which completes the proof of the Theorem.

Proof of Corollary 1. By our Theorem, it follows from (3) that

$$
\begin{aligned}
N & \geq \frac{|A||B||C||D|}{p}-(|A||B||C||D|)^{1 / 2} p^{1 / 2} \\
& =\frac{|A||B||C||D|^{1 / 2}}{p}\left((|A||B||C||D|)^{1 / 2}-p^{3 / 2}\right)>0 .
\end{aligned}
$$

\section{References}

[1] P. Erdős and A. Sárközy, On differences and sums of integers, I, J. Number Theory 10 (1978), 430-450.

[2] P. Erdös and N. H. Shapiro, On the least primitive root of a prime, Pacific J. Math. 7 (1957), 861-865.

[3] J. Friedlander and H. Iwaniec, Estimates for character sums, Proc. Amer. Math. Soc. 119 (1993), 365-372.

[4] M. Z. Garaev and F. Luca, On a theorem of A. Sárközy and applications, J. Théor. Nombres Bordeaux, to appear. 
[5] K. Gyarmati, On a problem of Diophantus, Acta Arith. 97 (2001), 53-65.

[6] A. Sárközy, On the distribution of residues of products of integers, Acta Math. Hungar. 49 (1987), 397-401.

[7] I. Schur, Über die Kongruenz $x^{m}+y^{m} \equiv z^{m}(\bmod p)$, Jahresber. Deutschen Math. Verein. 25 (1916), 114-117.

[8] I. M. Vinogradov, The Method of Trigonometrical Sums in the Theory of Numbers, Interscience, London 1954 (translated from the Russian, the Russian original appeared in 1947).

Department of Algebra and Number Theory

Eötvös Loránd University

Pázmány Péter sétány $1 / \mathrm{c}$

H-1117 Budapest, Hungary

E-mail: sarkozy@cs.elte.hu 